\title{
Mobile robotic cart development to support home oxygen therapy patients: Study on handling interface for mobile robotic cart similar to wheeled walker
}

\author{
Masatsugu Iribe ${ }^{*}$, Naoto Maeta', Gen Endo ${ }^{2}$,Toshio Takubo ${ }^{3}$, Mineko Ohira $^{4}$ and Tetsuya Kinugasa ${ }^{5}$
}

\begin{abstract}
We propose a motion control interface system for a mobile robotic cart to support Home Oxygen Therapy (HOT) patients when they go out. Our goal is to realize a mobile robotic cart similar to a wheeled walker that carries oxygen devices in front of patients, because HOT patients whose physical strength has especially waned would like to use such a cart according to the results of our questionnaire given to patients. The proposed handling interface of the mobile robotic cart adopts simple mechanisms and commonly used force sensors to sense only pushing and pulling forces from the user. We also apply a two-degrees-of-freedom (2 D.O.F.) control system to control the velocity and response time of the robotic cart. The feedforward controller of the 2 D.O.F. control system, which is an impedanceadjustable element, is expected to be used not only to adjust the response time but also to compensate for interference between the forces applied by the user and the robotic cart during operation. In this study we evaluated the effectiveness of our proposed handling interface mechanisms and control system by performing several experiments on a prototype robotic cart, and then we carried out user tests on some HOT patients and received a positive evaluation of the robotic cart's performance.
\end{abstract}

Keywords: Home oxygen therapy, Mobile robot, Handling interface mechanism, 2 D.O.F. control system

\section{Background}

Home oxygen therapy (HOT) is a medical treatment for patients who suffer from lung problems such as chronic obstructive pulmonary disease (COPD), and it is thought that about 160,000 patients are currently receiving HOT treatment in Japan [1]. HOT patients must receive highly concentrated oxygen to maintain a constant level of oxygen in their blood, thus patients must carry a portable oxygen tank when they go out as shown in Fig. 1. In particular COPD patients are recommended to perform moderate exercise, i.e., walking outdoors, to maintain their physical strength, so they must use portable oxygen tanks. However,

\footnotetext{
*Correspondence: iribe@osakac.ac.jp

${ }^{1}$ Department of Electro-Mechanical Engineering, Osaka ElectroCommunication University, 18-8 Hatsu-cho, Neyagawa-shi, Osaka 572-8530, Japan

Full list of author information is available at the end of the article
}

the total mass of the portable oxygen equipment, which includes a portable oxygen tank, an oxygen saver (which is a flow controller), and a carry cart, is about $5 \mathrm{~kg}$, which is sufficient to discourage patients from going out. In a questionnaire given to $88 \mathrm{HOT}$ patients, 55 respondents $(62.5 \%)$ answered that a reason for avoiding going out is the problem of the portable oxygen equipment. In addition 36 respondents, who represented the problem of the portable oxygen equipment above, also commented that the weight of the portable oxygen equipment is a problem [2].

With this background, we have proposed and developed mobile robotic cart prototypes with Hyper-Tethertype operational interface to carry oxygen equipment and follow HOT patient to encourage the patient to go out. We also have shown the effectiveness of such a robotic cart for carrying portable oxygen equipment in several user tests [3-5]. On the other hand, there are currently several types of oxygen equipment carriers that can 


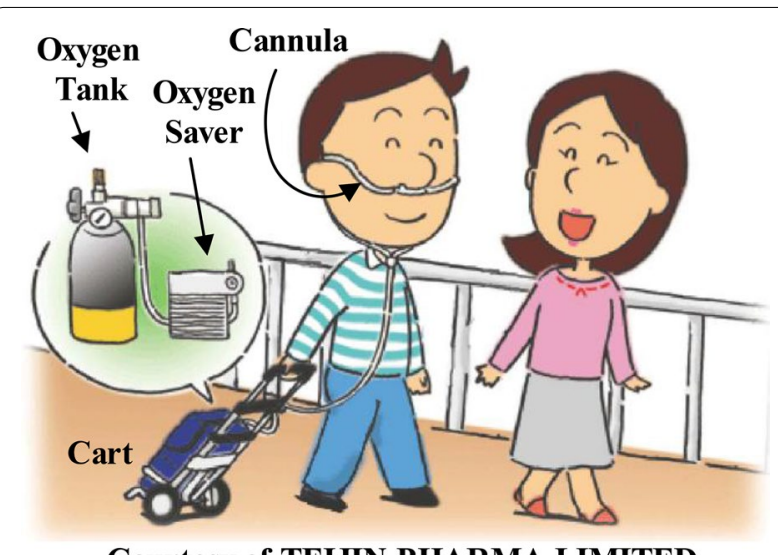

Courtesy of TEIJIN PHARMA LIMITED

Fig. 1 Home oxygen therapy (HOT) patients must use a set of oxygen equipment when they go out

accompany HOT patients. Thus, it may be possible to find out a suitable type of robotic cart without a HyperTether-type interface to meet the needs of patients.

In this paper, on the basis of the results of a questionnaire investigating the needs of HOT patients, we propose a new type of mobile robotic cart that moves in front of the users similarly to a wheeled walker that can carry a set of portable oxygen equipment. Especially, as part of the development, we study the operation of the handling interface mechanism and control system of the mobile robotic cart.

\section{Methods}

\section{Needs survey to identify feasible style of mobile robotic} cart

There are several types of conveyance cart for carrying oxygen equipment for HOT patients such as the wheeled carry-on type and the wheeled walker type. By considering the current apparatus, we can probably find other types of mobile robotic cart that satisfy patient needs in addition to the Hyper-Tether-type robotic cart mentioned in the previous section. Naturally, it is important to survey the needs of patients accurately before developing a robotic cart for HOT patients.

We thus designed a questionnaire for HOT patients to determine needs regarding the moving position and operation method of a mobile robotic cart. We carried out the first survey in Tokyo in June 2012 in cooperation with the NPO J-BREATH, which is a patient-based for people with respiratory disabilities, and then we carried out a second survey in Nagano in September 2012 in cooperation with HOKUSHIN Flying Disk Club, which is a patient-based group that aims to improve the quality of life of HOT patients through a flying disk game [6]. The questionnaire is shown in Fig. 2, and the results are shown in Fig. 3.
What type of mobile robotic cart do you prefer to use ?

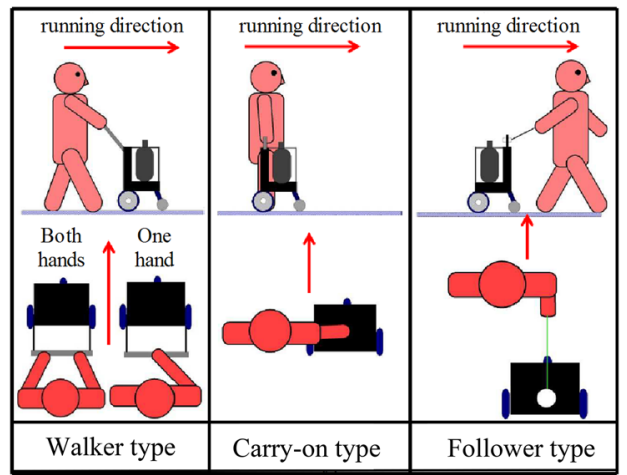

Fig. 2 The questionnaire contents to the HOT patients

The results in Fig. 3a show that patient preferences do not concentrate on a particular type of cart. However, examining the mutual relations among the preferred types of cart and the average time spent going out per week, which indicates the activeness of the patients, we can confirm that the patients who do not go out much tend to prefer walker type as shown in Fig. 3b.

On the basis of these results, we attempted to realize a mobile robotic cart that conveys oxygen equipment and moves in front of the user. Particularly, in this study, we attempted to establish a motion control method for a mobile robotic cart that moves in front of the user and conveys oxygen equipment, similarly to the wheeled walker shown in Fig. 4, which is a current oxygen equipment carrier supplied from a medical device manufacturer.

\section{Concept of the proposed mobile robotic cart}

In this study, on the basis of the questionnaire, we attempt to realize a mobile robotic cart which moves in front of the user and convey the oxygen equipment. We often find researches on the robotic cart, which is similar to our proposed robotic cart, in the field of medical and welfare. The researches usually aim to support the elderly or disability people who have difficulty with ambulation $[7,8]$. Thus, for the purpose of implementation of the robotic cart, the researches adopt the operating forces, which are applied to the handles, to control the motion of the robotic cart for supporting users ambulation [9-12].

On the other hand, our goal is to reduce the transport loads of HOT patients, not to support the elder and the people with difficulty of walking. Therefore, at first, it is important for the robotic cart to be able to follow the HOT patient accurately and stop correctly, same as the past researches. In addition, the robotic cart must stop its operation to avoid malfunction, not to continue its conveyance operation, 


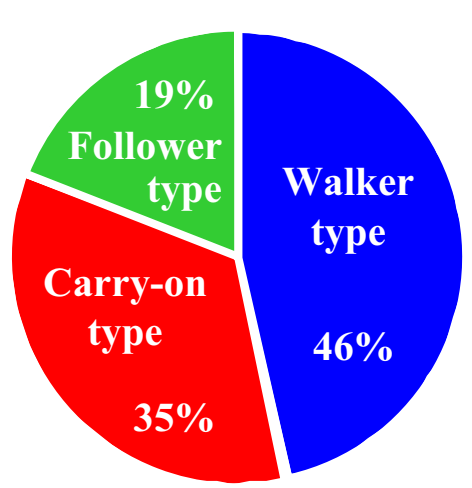

a

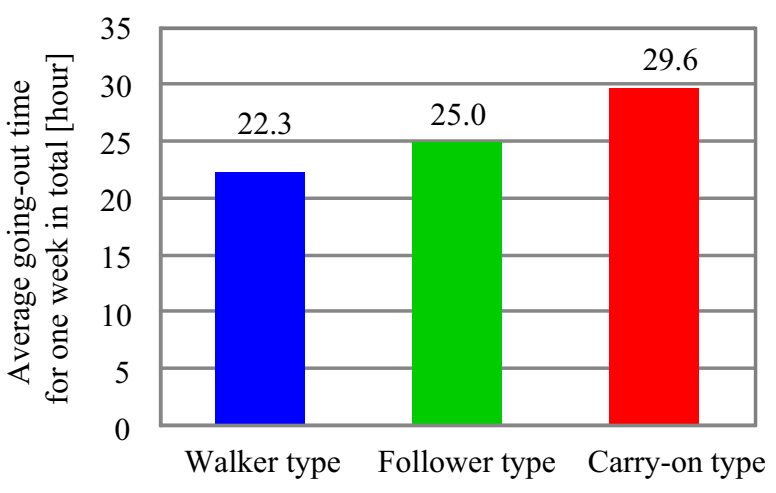

b

Fig. 3 Questionnaire results from HOT patients. There is a correlation between the preferred type of cart and the average time spent going out per week. a Questionnaire result about preferred cart type, valid response 27, multiple answers are allowed. $\mathbf{b}$ Correlation between the patients average time spent going out per week and preferred cart type

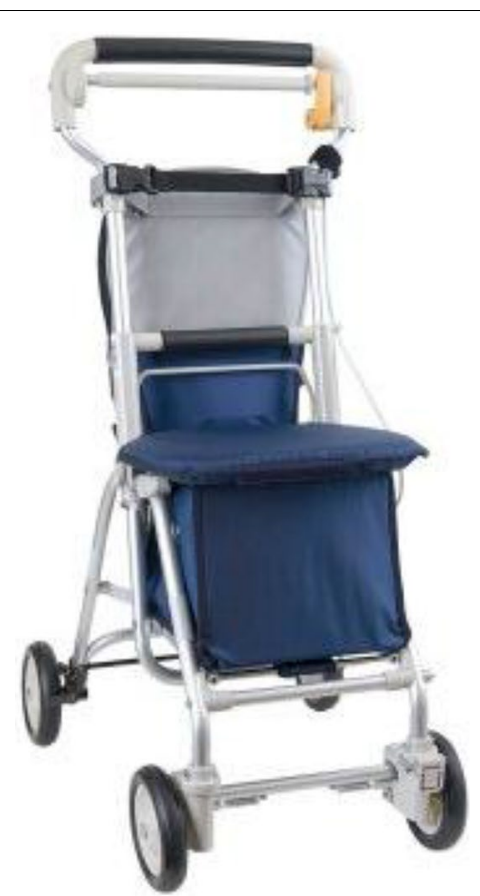

Fig. 4 Current Oxygen equipment carrier like wheeled walker. Courtesy of Teijin Pharma Limited

when the motion of grasping or leaning on the handle, which is not normal operation, occurs. To realize such the function, we need the high-performance control system that can also adjust the response property by itself, and also need the handling interface mechanism that can sense the input forces correctly. We, thus, propose and develop a filter type two-degrees-of-freedom control system for the controller and new interface mechanism for the operational handle.
We show a concept of our proposed mobile robotic cart in Fig. 5. We adopt two passive wheels and two drive wheels for the robotic cart as shown in the Fig. 5. Users hold the handle grips installed on the mobile robotic cart to control the cart's motion. The cart's motion is controlled by the sum of the left and right drive-wheel velocities and the ratio of the two drive-wheel velocities, which are calculated from the pushing forces of the handle grips. The reference velocities of the two drive wheels are calculated by

$$
\left\{\begin{array}{l}
V_{R-r e f}=K_{S}\left(F_{R}+F_{L}\right)-K_{T}\left(F_{R}-F_{L}\right) \\
V_{L-r e f}=K_{S}\left(F_{R}+F_{L}\right)+K_{T}\left(F_{R}-F_{L}\right)
\end{array},\right.
$$

where $F_{R}$ and $F_{L}$ are the pushing forces sensed on the right and left handle grips respectively, which are positive in the forward direction. $V_{R-r e f}$ and $V_{L-r e f}$ are the reference velocities of the velocity feedback control system for the right and left drive wheels. $K_{S}$ and $K_{T}$ are coefficients representing the sensitivity for straight motion and turning motion, respectively. Users will be able to adjust these coefficients according to their needs.

\section{Our proposed handling interface mechanism}

For our proposed mobile robotic cart development, we attempted to develop a handling interface and a motion control method for a mobile robotic cart that can be operated similarly to a wheeled walker type conveyance cart as shown in Fig. 5. When using conveyance carts similar to our proposed robotic cart, users often lean on the cart because its shape is easy to lean on. Moreover, the user may often grip and twist the handle grips unconsciously. It is therefore assumed that these unconscious actions will generate unintentional operating forces, causing the 


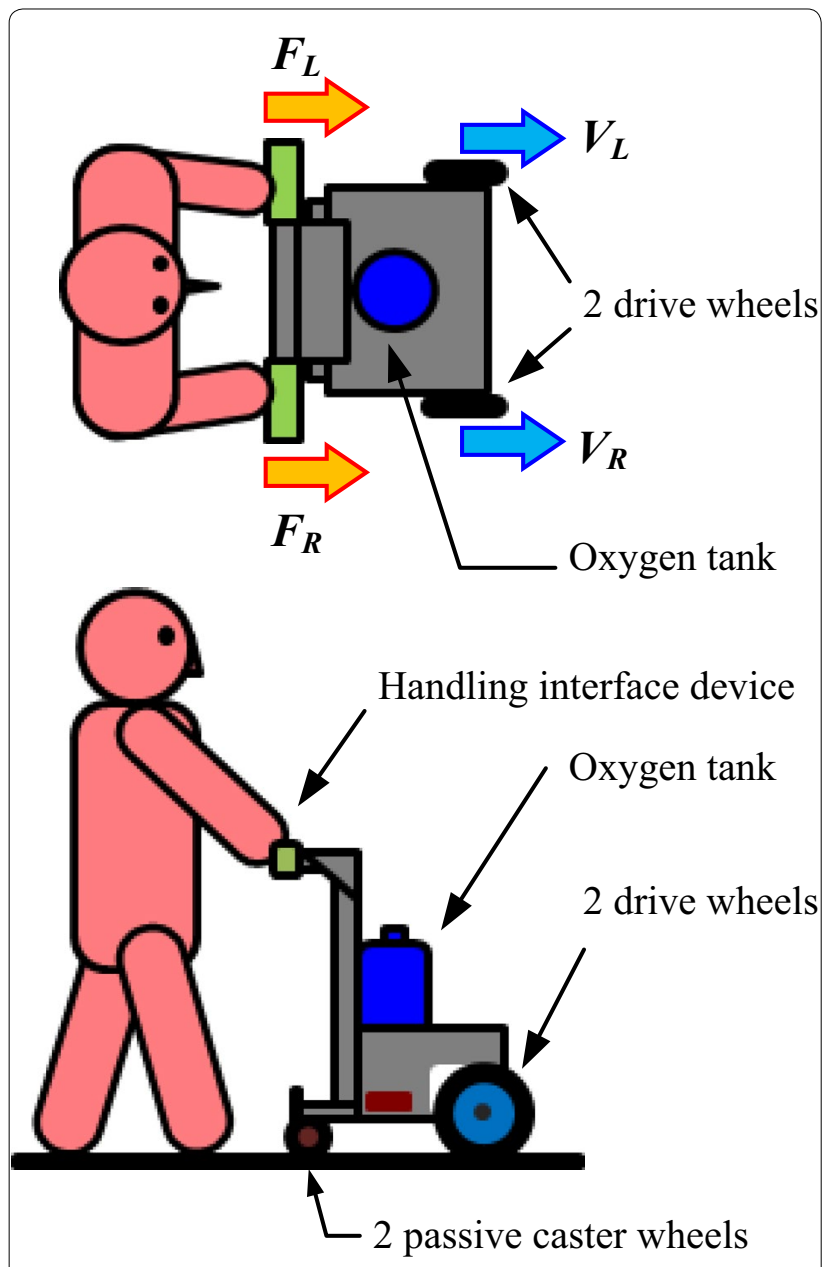

Fig. 5 Our proposed mobile robotic cart similar to a wheeled walker

erroneous motion of the mobile robotic cart. As a countermeasure against this problem, we adopt the handling interface shown in Fig. 6.

In the proposed interface structure, a solid grip comprising a square aluminum pipe is fixed to cover the force sensors fixed on the right and left ends of the handle pillar. There are a total of six force sensors fixed to the right and left ends of the handle pillar. It is designed so that the internal dimension of the solid grip cover in the transverse direction is larger than the outer dimension of the handle pillar to allow $0.5 \mathrm{~mm}$ clearance. There are also thin force sensors fixed by polyvinyl tape between the handle pillar and the solid grip cover.

The force sensor fixed to the back of the handle pillar is named the forward force sensor (FFS) which senses the pushing force; the force sensor fixed to the front of the handle pillar part is named the backward force sensor (BFS), which senses the pulling force; and the force sensor fixed to the top of the pillar is the downward force sensor (DFS), which senses the force applied from above. Each type of sensor is fixed to the right and left ends of the handle pillar. Since the handle grip adopts this mechanism, $F_{L}$ and $F_{R}$ in Eq. (1) are calculated as the difference between the values obtained by the FFS and BFS as follows:

$$
F_{L}=F_{L_{-} F F S}-F_{L_{-} B F S}, \quad F_{R}=F_{R_{-} F F S}-F_{R_{-} B F S} .
$$

The mechanisms for detecting the intended actions of the users are shown in Fig. 7. When the user does not the hold handle grips, the force sensors do not sense the forces, as shown in Fig. 7 (1). When the user moves forward or backward, the handle grip is pressed obliquely from above by the user, as shown in Fig. 7 (2), and the downward force that is sensed by the DFSs becomes greater than the set threshold value. At the same time, the FFSs or BFSs is also pressed and the pushing or pulling force applied to the handle grip is sensed. When a downward force and a forward or backward force are simultaneously detected, as described above, we can determine the motion of the robotic cart for forward or backward.

The unintended motion such as the grasping sometimes causes misdetection because the force sensors fixed in the handle grip are pressed at the same instant by the grasping motion. Our proposed handling interface prevents such misdetection due to the grasping motion, which causes a malfunction, because the solid grip cover blocks the gripping forces, as shown in Fig. 7 (3). The twisting motion also sometimes causes similar misdetection. However, when the user twists the handle grip, as shown in Fig. 7 (4), the DFSs do not come in contact (or only come in slight contact) with the solid grip cover because of our proposed mechanical structure. Therefore, when the user twists the handle grip, the downward force sensed by the DFSs becomes smaller than the set threshold value. On the other hand, the FFSs and BFSs are pressed at the same time; thus, the sensors sense the pushing or pulling force applied to the handle grip as shown in Fig. 7 (4). Although the forces are detected, we can prevent the erroneous motion of the robotic cart because the downward force is smaller than the set threshold value.

In the case that the user only leans on the robotic cart without operating the handle grips, all of the user's weight becomes a load detected by the DFSs; thus, no signal is sensed by the FFSs or BFSs. Therefore, we can ensure that the robotic cart remains stationary by monitoring the value from the DFSs.

We, then, carried out some measurement experiments to confirm the effectiveness of our proposed handling interface. In the measurement experiments, the participant (normal male age 23) operated the handle grip of the prototype robotic cart which is shown in Fig. 10. We measured the force signals detected by the FFS, BFS, and DFS when the participant moved in a straight line and only twisted the handle grip in the forward and backward directions. 


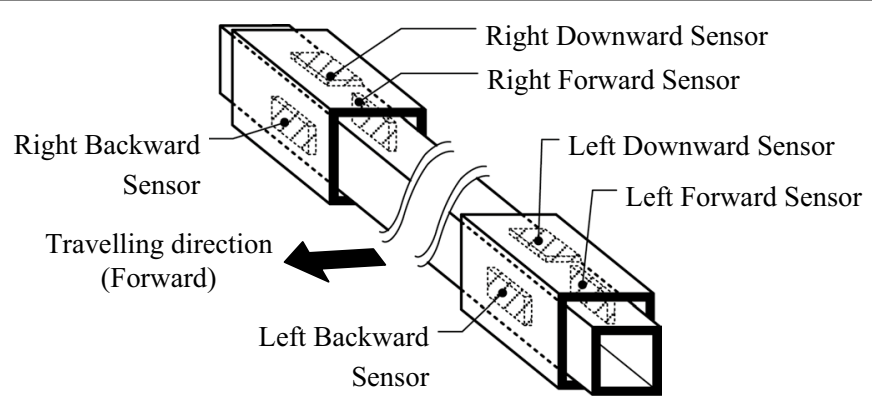

a

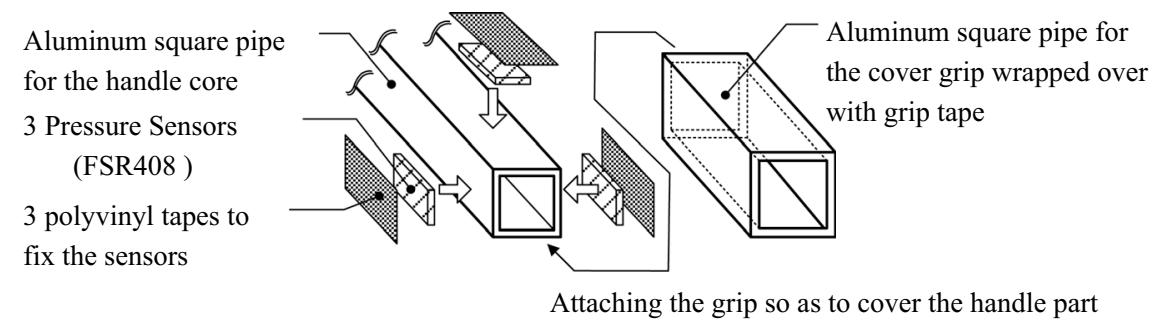

b

Fig. 6 Proposed handling interface mechanism. a Layout of force sensors in the handling interface mechanism. $\mathbf{b}$ Detailed structure of the handling interface mechanism and force sensors' mounting

(1) Neutral

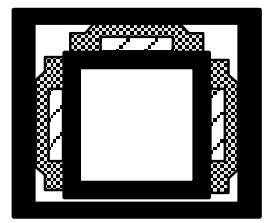

(3) Non-reactive Operation (Grasp)

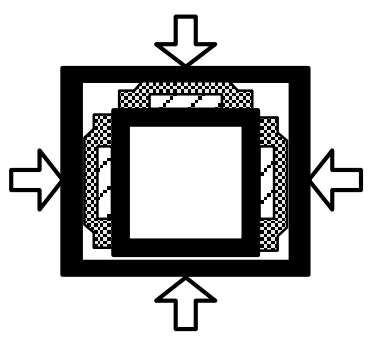

(2) Normal Operation

(Backward)

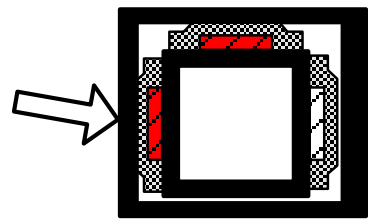

(Forward)

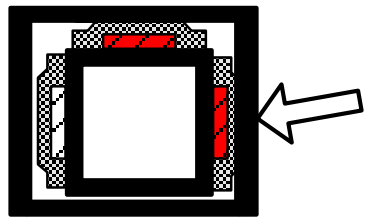

(4) Non-reactive Operation

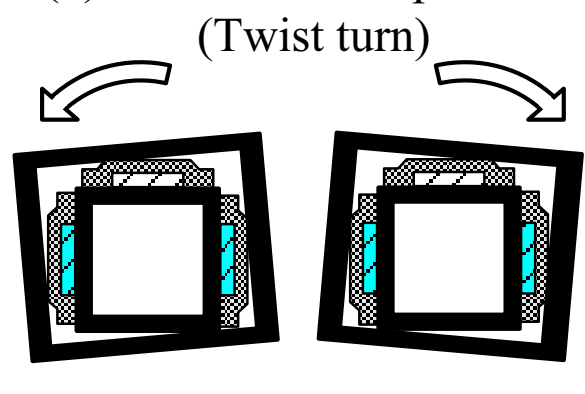

Fig. 7 Operational force detection and estimation of the users' behavioral intention 
Figure 8 shows the experimental results for the right side handle grip. We found that the force detected by the DFS during forward and backward movement was more than $4.0 \mathrm{~N}$ as shown in Fig. 8a, b. We also found that the force detected by the DFS during twisting motion was less than $2.0 \mathrm{~N}$ as shown in Fig. 8c, d. On the basis of these results, the threshold value of the DFS was set to $2.0 \mathrm{~N}$, which was used to predict the intended movement of the user. By adopting the handling interface shown in Fig. 6, it is possible to predict the user's intended movement as well as three operations that cause malfunction: grasping, twisting, and leaning on the robotic cart.

\section{Concept of control system design}

The mobile robotic cart proposed in this study adopts the velocity control system to control the velocities of the left and right drive wheels according to the operating forces applied to the handle grip by the user as shown in Fig. 5 .

It is of course necessary for the velocity control system to rapidly respond to the user. On the other hand, the assumed users of the robotic cart are HOT patients; thus parameters such as the maximum velocity and acceleration should be adjusted according to the user's age, physical strength, and size. Therefore, in the control system of the robotic cart, a new function is required to adjust the maximum velocity and acceleration for each user in addition to the general characteristics of the controller such as the steady state and tracking performances.

To meet these requirements, we propose a filter type two-degrees-of-freedom control system whose input signal is the pushing force $F_{R}$ and $F_{L}$, and whose output signal is the velocity $v_{R}$ and $v_{L}$. Figure 9 shows a block diagram of the control system; $F_{R}$ and $F_{L}$ are Forces from the user which are applied to the right and left handle grips, $V_{R-r e f}$ and $V_{L-r e f}$ are Reference velocities calculated by Eq. (1), $\hat{V}_{R-r e f}$ and $\hat{V}_{L-r e f}$ are Reference velocities passed through the feedforward controller $C_{1-L}(s)$ and $C_{1-R}(s)$, $C_{1-R}(s)$ and $C_{1-L}(s)$ are Transfer functions of the feedforward controller which consist of first-order lag element, $C_{2-R}(s)$ and $C_{2-L}(s)$ are Transfer functions of the feedback controller which are PID controller, $G_{R}(s)$ and $G_{L}(s)$ are Transfer functions of the control object which is the mechanical system of the robotic cart.

We adopt PID controller for $C_{2-R}(s)$ and $C_{2-L}(s)$, which determines the velocity feedback control characteristic,

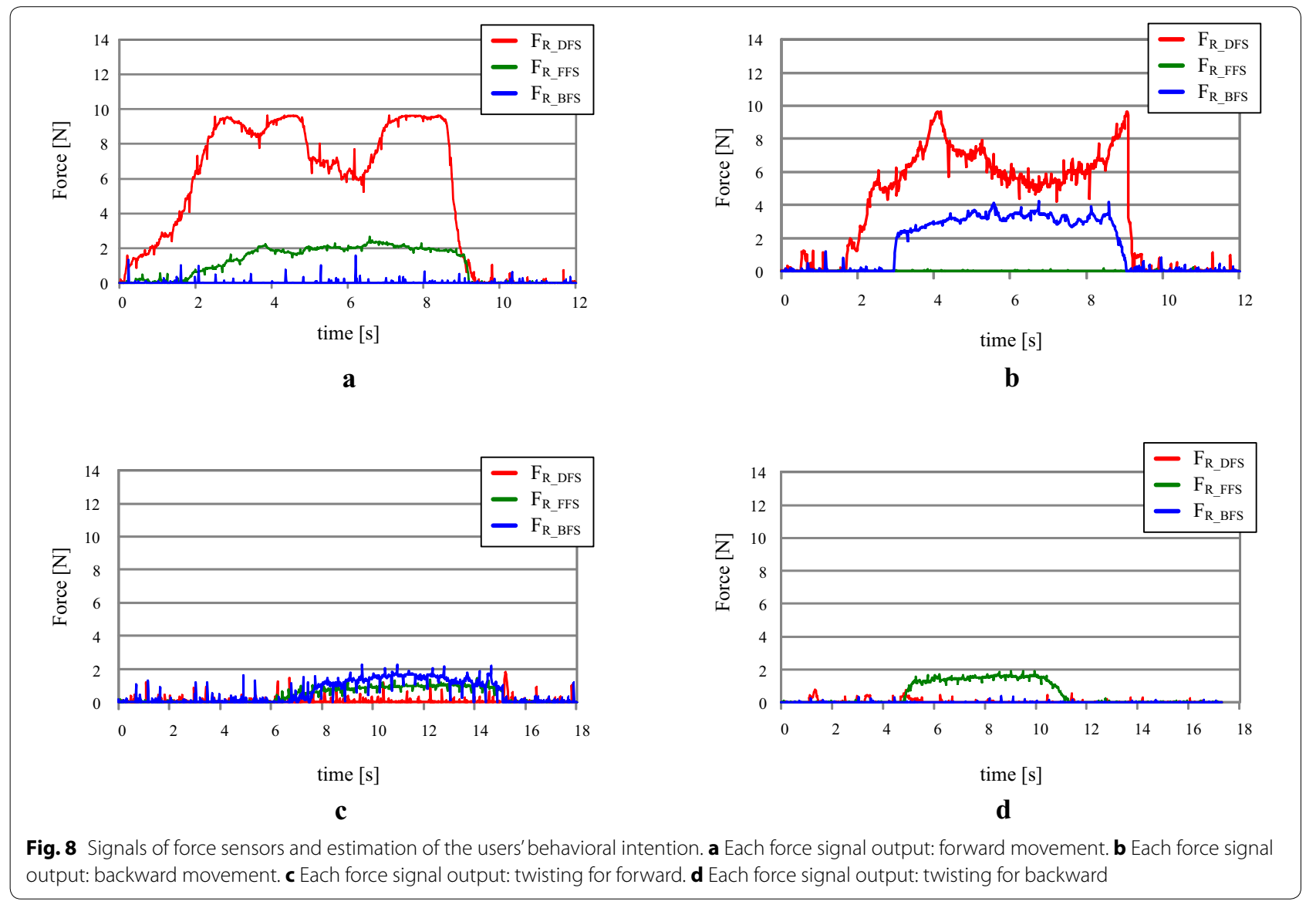




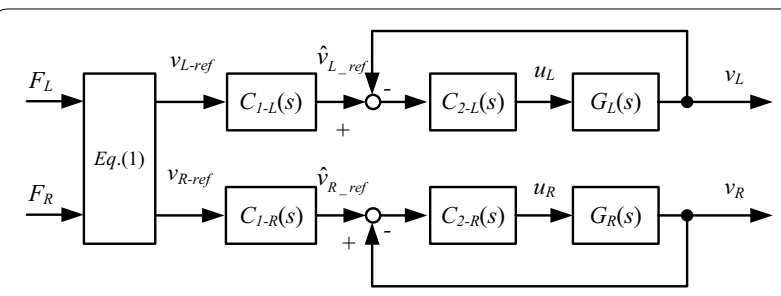

Fig. 9 Control system block diagram of our proposed robotic cart

and also adopt a first-order lag element filter for $C_{1-R}(s)$ and $C_{1-L}(s)$, which determines the feedforward control characteristics of the input and output responses for our proposed control system shown in Fig. 9. The feedforward controllers $C_{1-R}(s)$ and $C_{1-L}(s)$ adjust the maximum velocity and the start-up response time of the robotic cart, in other words, they act as elements to adjust the mechanical impedance. We describe the specific contents of the elements used to configure the control system as follows.

The parameters $K_{1-L}$ and $K_{1-R}$ in Eq. (3) are used to adjust the maximum velocity of the left and right drive wheels respectively. The parameters $T_{1-L}$ and $T_{1-R}$ in Eq. (3) are the start-up time constants used to adjust the start-up response of the robotic cart. The parameters $K_{2 L_{-} P}, K_{2 L_{-} l}, K_{2 L_{-} D}, K_{2 R_{-} P}, K_{2 R_{-}}$, and $K_{2 R_{-} D}$ in Eq. (4) are the control gains of the PID controller. The parameters $M_{C}$, $R_{W}$ and $D_{C}$ are the mass of the robotic cart, the radius of the drive wheel, the coefficient of dynamic friction, respectively.

$$
\begin{aligned}
& C_{1-R}(s)=\frac{K_{1-R}}{T_{1-R} s+1}, \quad C_{1-L}(s)=\frac{K_{1-L}}{T_{1-L} s+1} \\
& C_{2-R}(s)=K_{2 R_{-} P}+s K_{2 R_{-} D}+\frac{K_{2 R_{-} I}}{s}, \\
& C_{2-L}(s)=K_{2 L_{-} P}+s K_{2 L_{-} D}+\frac{K_{2 L_{-} I}}{s} \\
& G_{R}(s)=\frac{K_{T_{-} L}}{R_{W}} \frac{1}{0.5 M_{C} s+D_{C}}, \\
& G_{R}(s)=\frac{K_{T_{-} R}}{R_{W}} \frac{1}{0.5 M_{C} s+D_{C}}
\end{aligned}
$$

We first design the feedback controller $C_{2}(s)$ whose input is $\hat{v}_{\text {ref }}$ and output is $v$ because $C_{2}(s)$ determines the stability of the velocity feedback control system. After designing $C_{2}(s)$, we design the feedforward controller $C_{1}$ $(s)$ so that the moving velocity and start-up response can be tailored to individual users. We found that the control system satisfies its designed purpose when adopting this configuration.
Evaluation of control system using a prototype robotic cart Here we evaluate the feasibility and effectiveness of our proposed control system by performing several experiments using the laboratory prototype mobile robotic cart shown in Fig. 10, whose main specifications are given in Table 1. This robotic cart was designed for evaluation tests in an indoor environment or on a flat road surface. The handle grips of the prototype have the structure shown in Fig. 6, which detect the operational forces applied by the user. The reference velocities of the right and left drive wheels are calculated from the detected operational forces using Eqs. (1) and (2), and the motion of the prototype is controlled by the control system described in Fig. 9 on the basis of the calculated reference velocities. We can adjust the handle height from 765 to $945 \mathrm{~mm}$ in seven steps, allowing the prototype robotic cart to fit the height of the user.

Figure 11 shows a block diagram of the hardware of our proposed control system. A microcomputer mounted on an SH2 Tiny Controller (Hi-bot Inc.) controls the entire system of the prototype robotic cart. The microcomputer detects the output voltages from FSR408 force sensors (Interlink Electronics Inc.), and variable resistors are set to adjust the parameters of the feedforward controller $C_{1}$ (s) through an $\mathrm{AD}$ converter circuit. The microcontroller also counts the pulse signal outputs from rotary encoders attached to the wheel drive motors to detect the angular velocities of the drive wheels through a pulse counter circuit embedded in the microcomputer. From the input signals from the force sensors, variable resistors and rotary encoders, the velocity controller shown in Fig. 9 calculates the PWM duty signals for the drive motors, and then the controller controls the motion of the prototype robotic cart.

Figure 12 shows the step response of the prototype robotic cart when a constant value is given to the controller as a reference velocity programmatically (not by the user). Figure 12a shows the step response from the input values $\hat{V}_{R-\text { ref }}$ and $\hat{V}_{L-r e f}$ to the output values $V_{R}$ and $V_{L}$ without the feedforward controller $C_{1}(s)$. Figure $12 \mathrm{~b}$ shows the step response trajectories of the right drive wheel when the time constants $T_{1-R}$ and $T_{1-L}$ of the feedforward controllers are changed experimentally. The experimental result shown in Fig. 12a indicates that the velocity feedback controller $C_{2}(s)$ can ensure that the response characteristic of each drive wheel is equal and stable. Also, the experimental results shown in Fig. 12b indicate that the feedforward controller $C_{1}(s)$ can adjust the start-up response between the input and output signals. In particular, it can adjust the start-up time constant from 0.05 to $2.0 \mathrm{~s}$. These results show that our proposed control system can act as a stable velocity feedback 


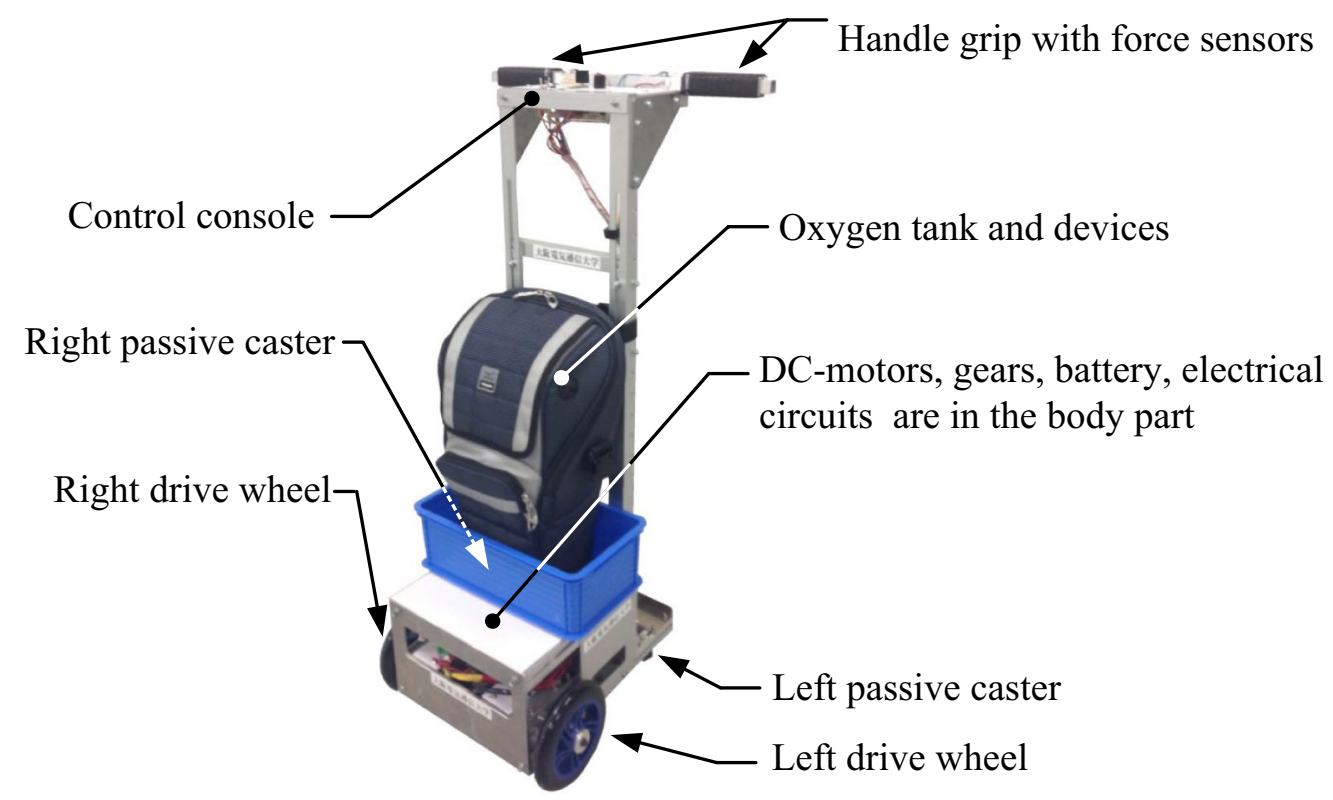

Fig. 10 Prototype robotic cart for evaluation

Table 1 The prototype robot's specification

\begin{tabular}{ll}
\hline Dimension $[\mathrm{W} \times \mathrm{D} \times \mathrm{H}]$ & $360 \times 370 \times 765 \sim 945[\mathrm{~mm}]$ \\
Weight & $8.5 \mathrm{~kg}$ (with batteries) \\
Power supply & Nickel metal hybride \\
& $12 \mathrm{~V} / 4200 \mathrm{mAh}$ \\
& Series connection \\
Actuation & DC-motor: TG-85R-SU \\
& Reduction ratio: $1 / 69.14$ \\
$\mathrm{CPU}$ & $\mathrm{SH} 2 / 7047(\mathrm{CLK} / 44.2 \mathrm{MHz})$ \\
\hline
\end{tabular}

controller and adjust the sensitivity between the inputs and outputs.

\section{Results and discussion}

\section{Test to evaluate design}

We carried out tests to evaluate our design. In the tests, the participant (normal male age 23) operated a commonly used wheeled walker and the prototype robotic cart, moving it in a straight line and turning it to the right and left. At the same time, we measured the operating forces applied to the handle grip to assess the presence of the power assistance. Figure 13 shows the experimental results using commonly used wheeled walker, and Fig. 14 shows the experimental results using the robotic cart.

As shown in Fig. 13, the total operational forces applied to both the left and right handles by the participant were a smaller about $20 \mathrm{~N}$ while moving in a straight line and turning right and left. On the other hand, we found that the total operational forces applied to the left and right handles of a prototype robotic cart were less than $10 \mathrm{~N}$ as shown in Fig. 14. We therefore found that our proposed robotic cart moves with a smaller applied force than that required for a current conveyance cart.

Then we investigated the power assistance effect of the prototype robotic cart when moving on a barrierfree slope. While the participant operated the prototype robotic cart on the barrier-free slope, as shown in Fig. 15, we compared the behaviors of the prototype robotic cart and a wheeled walker as a current conveyance cart at that time. The experimental results are shown in Fig. 16.

The average pushing force required to operate the wheeled walker was $15 \mathrm{~N}$, as shown in Fig. 16a, when the participant walked up the slope. On the other hand, the average operating force for the prototype robotic cart was $5.5 \mathrm{~N}$ as shown in Fig. 16b. Also, the operating forces added to the handle grips became zero when the participant temporarily stopped during the operation, as shown in Fig. 16b because there was no need to push the prototype robotic cart. In contrast, the pushing forces added to the wheeled walker remained over $5 \mathrm{~N}$ during the operation to maintain its stationary state as shown in Fig. 16a.

When the participant walked down the slope, the average operating force pushing the wheeled walker was $-5 \mathrm{~N}$ as shown in Fig. 16c. On the other hand, the average operating force of the prototype robotic cart was $5 \mathrm{~N}$ as shown in Fig. 16d. The operating forces added to the handle grips also became zero when the participant 


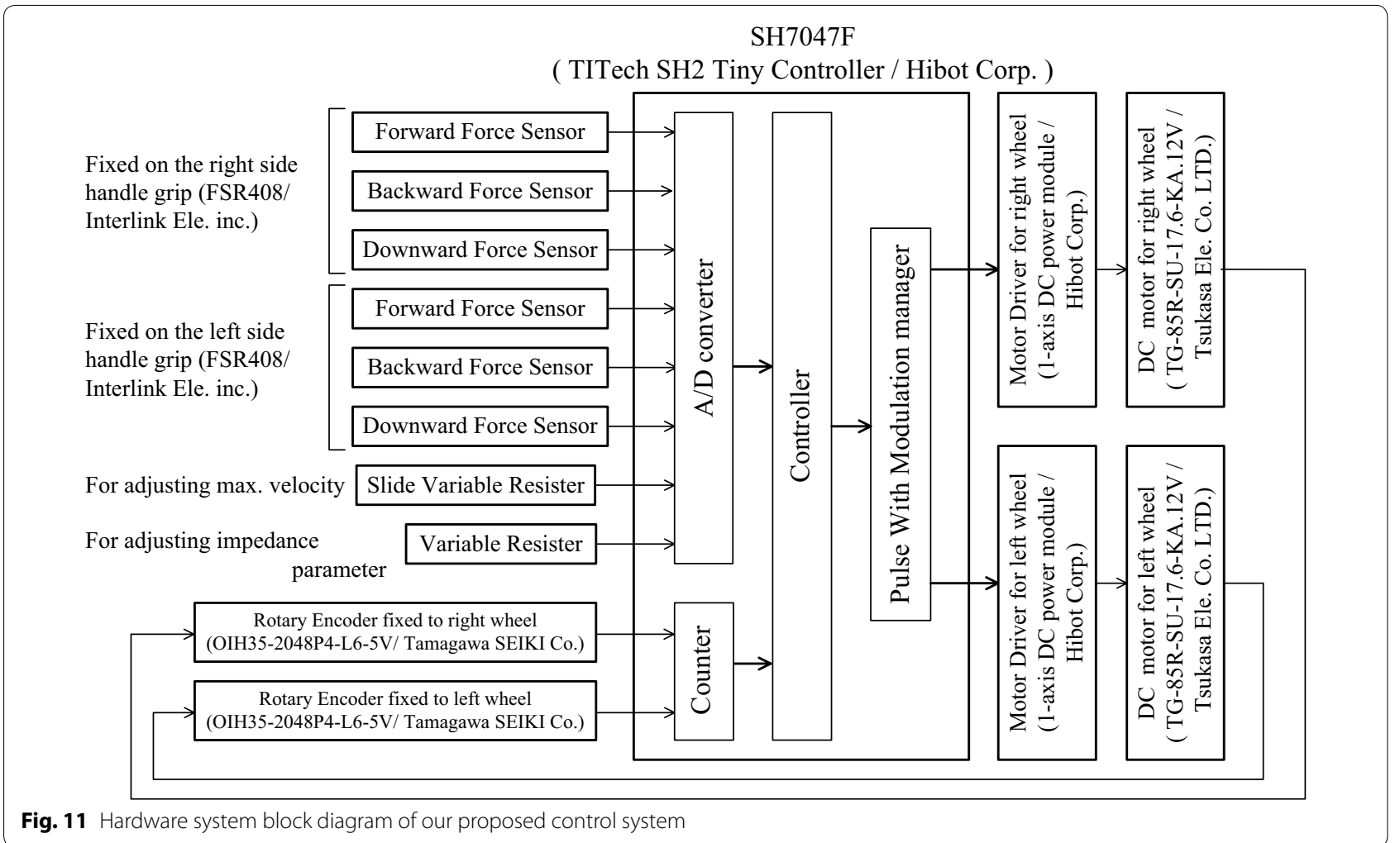

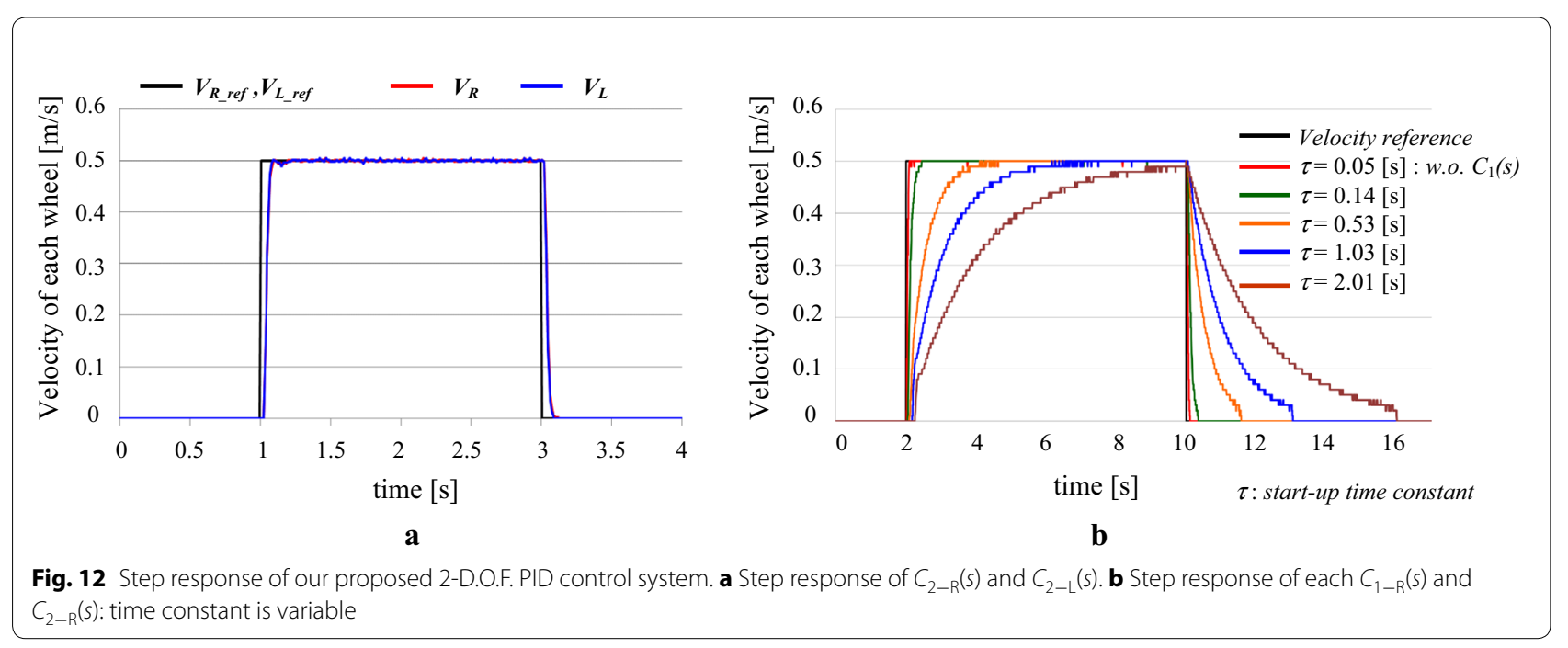

temporarily stopped during the operation as shown in Fig. 16d, because there was no need to hold the prototype robotic cart. In contrast, the pulling forces added to the wheeled walker remained under $-8 \mathrm{~N}$ during the operation to maintain the stationary stale as shown in Fig. 16c. These experimental results show that the prototype robotic cart moves according to only the pushing forces and that its motion is not affected by gravity.

From the above experimental results, we found out that the participant's operating forces are reduced by the prototype robotic cart. The results therefore clearly illustrate the power assistance effect of the prototype robotic cart. 

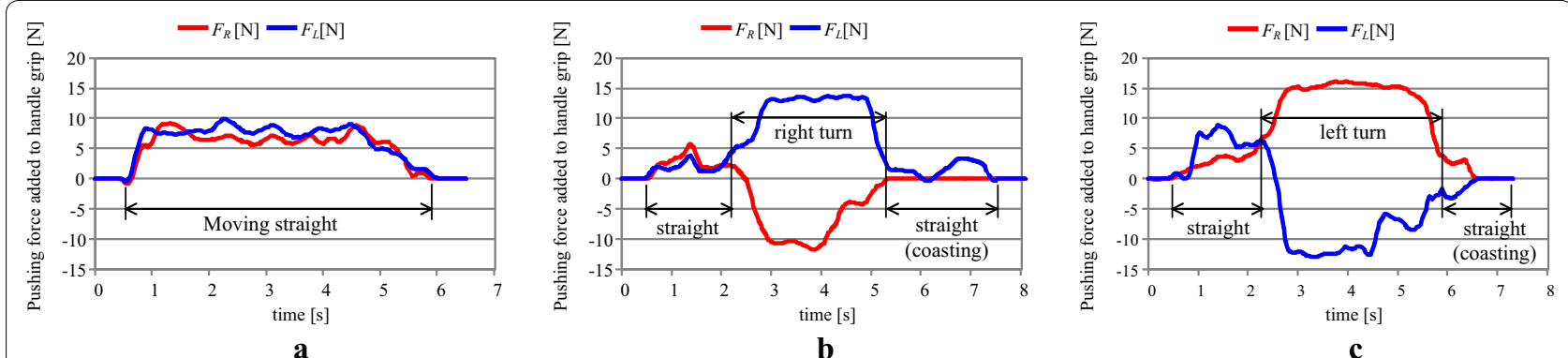

Fig. 13 Experimental results of handling force while the participant operated a commonly used wheeled walker. a Moving straight experiment. b Turning right experiment. c Turning left experiment

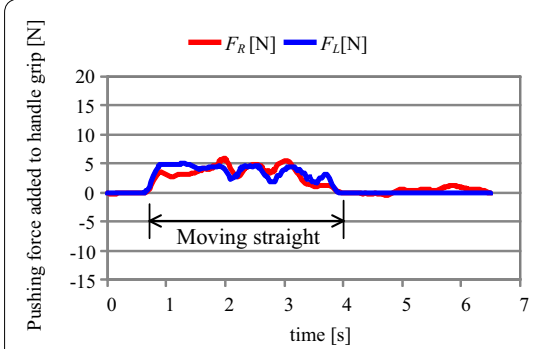

a

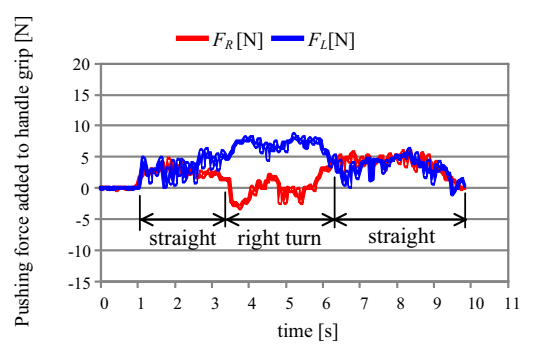

b

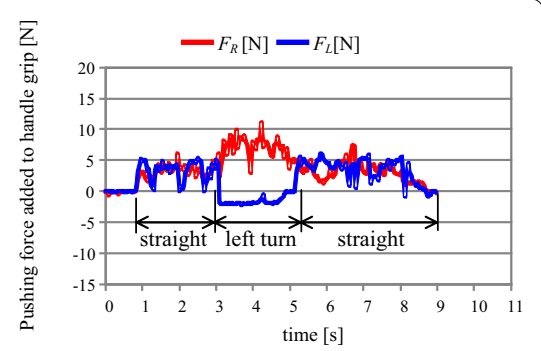

c

Fig. 14 Experimental results of handling force while the participant operated the prototype robotic cart. a Moving straight experiment. b Turning right experiment. c Turning left experiment

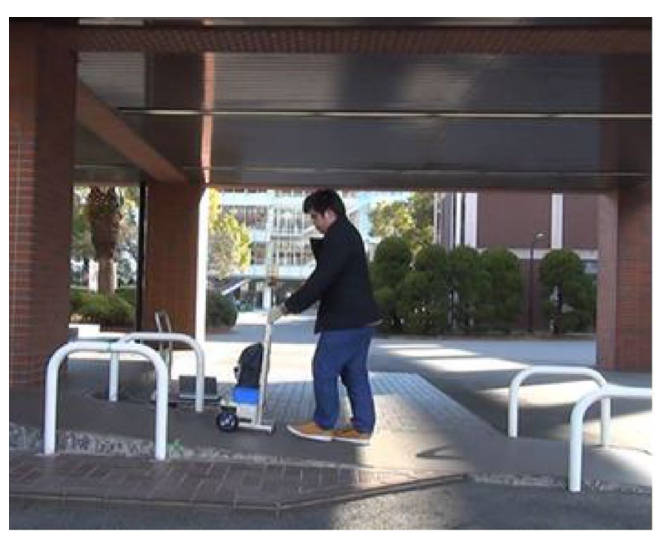

a

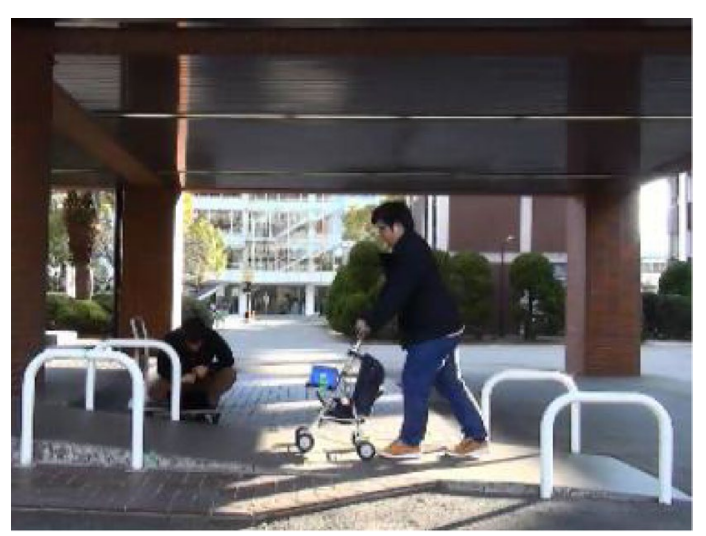

b

Fig. 15 Operational experiment on the barrier-free entrance slope by using the prototype robotic cart. a Walking up the slope by the prototype robotic cart. b Walking up the slope by a commonly used wheeled walker as a comparative sample

\section{Evaluation by HOT patients}

We next carried out a test in which HOT patients evaluated the operability of the robotic cart. Our experiments are approved by the ethical committee of Tokyo Institute of Technology (approval number 2012014 and 2013002).
We also obtained the consents of the participants for all experiments.

The test was carried out in cooperation with the patientbased Kiyose-KIBOEN group in Tokyo on September 26, 2013, as shown in Fig. 17a. In this user test, HOT patients 


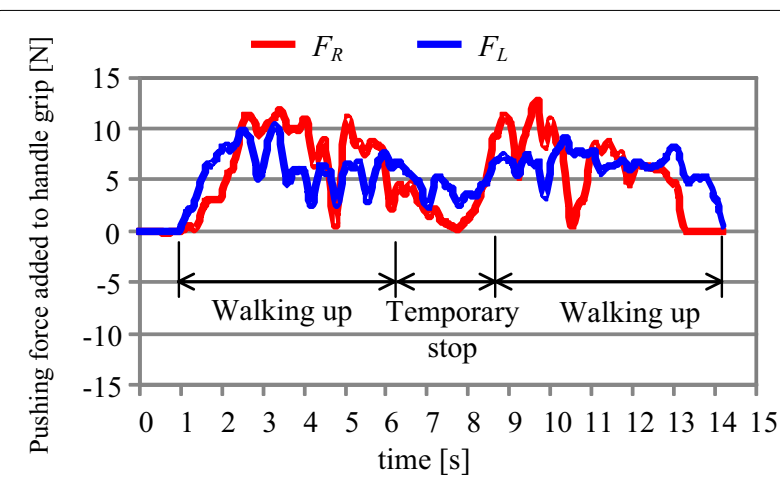

a

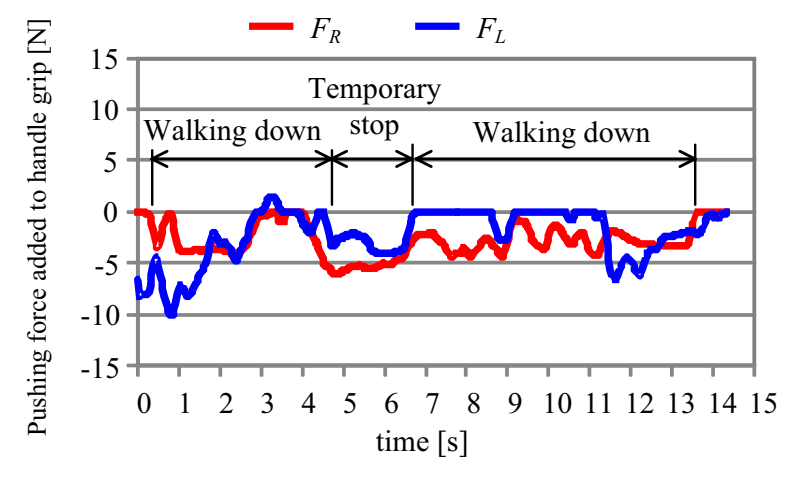

c

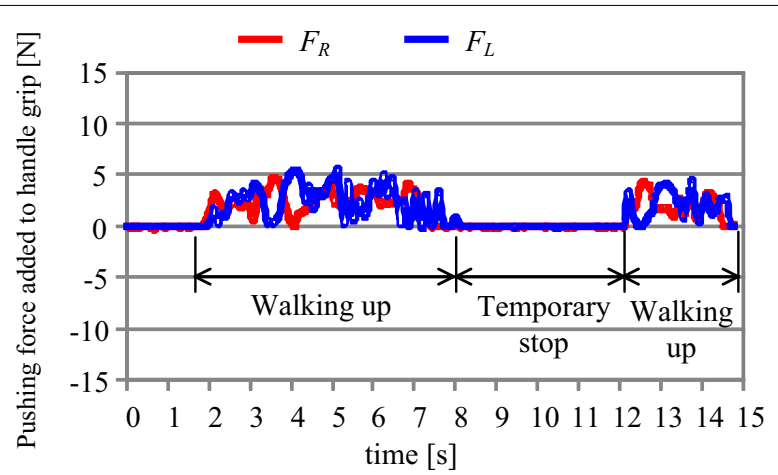

b

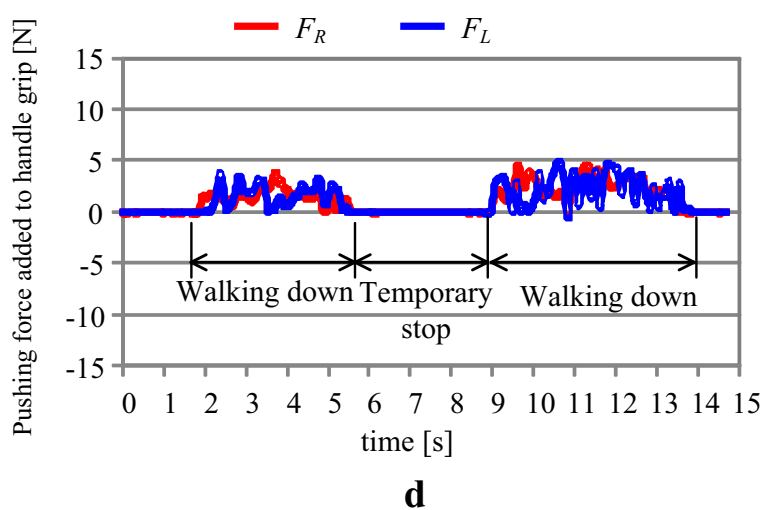

Fig. 16 Operational experiment results on moving up and down the barrier-free entrance slope. a Moving up the slope: wheeled walker. b Moving up the slope: prototype robotic cart. c Moving down the slope: wheeled walker. $\mathbf{d}$ Moving down the slope: prototype robotic cart

operated the prototype mobile robotic cart while moving forward in a straight line, after adjusting the handle heights that were decided by the participants. The participants were also allowed to adjust the setting parameters of the feedforward controller $C_{1}(s)$ to operate the prototype cart easily. The evaluation tests were carried out with an experimental assistant who took the participant's side and controlled a safety switch during the tests to prevent the emergency situation (Additional file 1).

We recorded the input and output signals when the participants operated the prototype mobile robot with their preferred parameters, and we also confirmed the leaning on and grasping detection worked correctly. Table 2 shows the average and maximum values of the pushing force of the participants in the experiment. Two HOT patients carried out the user test, one female age 74 (participant A) and one male age 69 (participant B).

The average pushing force of the users of a wheeled walker is $12-17 \mathrm{~N}$ typically [13]. Therefore, by comparing the results shown in Figs. 13 and 14, it can be seen that the average values in Table 2 are satisfying low. These results show the effectiveness of the mobile robotic cart for conveying oxygen equipment to support HOT patients.

\section{Evaluation by elderly people}

A test was also carried out involving the elderly people for comparison with the results for the HOT patients. This test was carried out in cooperation with NPO Corporation "Osaka Kohreisha Daigakko", which is a college of retired elderly people for their lifelong study, in Osaka on March 3rd, 2014, as shown in Fig. 17b.

In this test, 43 participants aged from 62 to 80 years operated the prototype mobile robotic cart while moving forward in a straight line similarly to in the previous test. The participants were also allowed to adjust the setting parameters of the feedforward controller $C_{1}(s)$ to operate the prototype cart easily. We recorded the input and output signals when the participants operated the prototype cart with their preferred parameters. In addition, we collected their impressions of the prototype by questionnaire. The results of the questionnaire (37 valid responses, 24 men, 13 women) are shows in Fig. 18 and indicate a favorable evaluation of the prototype robotic cart. 


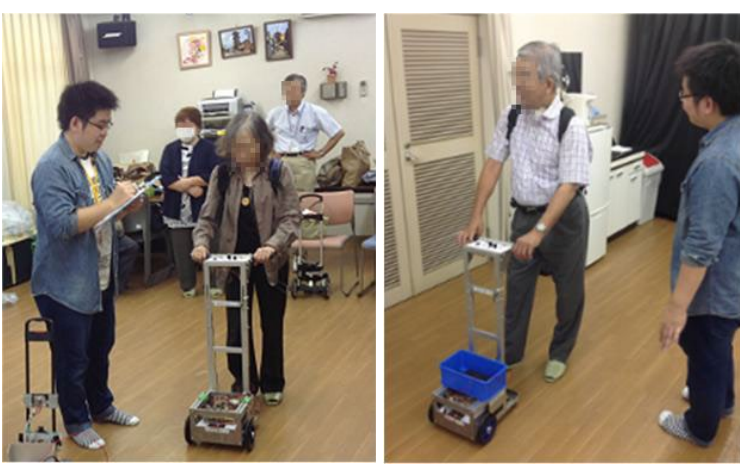

$\mathbf{a}$
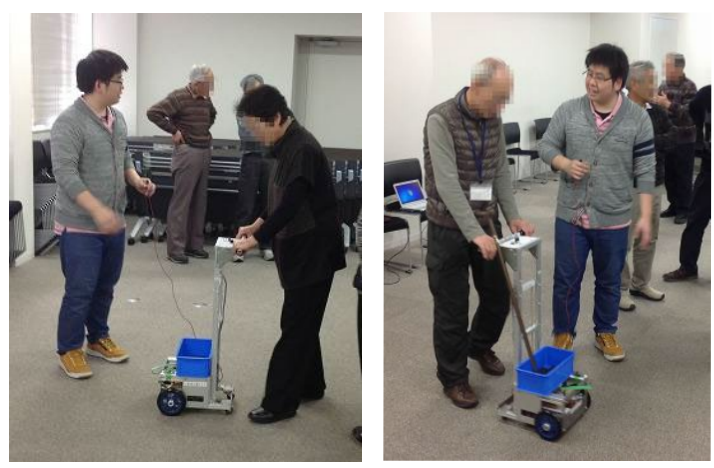

b

Fig. 17 User evaluation tests by HOT patients and elderly people. a Tests by HOT patients (Tokyo, 26th, Sep., 2013). b Tests by elderly people (Osaka, 3rd, Mar., 2014)

Table 2 Evaluation results by HOT patients

\begin{tabular}{lllllll}
\hline & \multicolumn{2}{l}{ Participant A } & & \multicolumn{2}{l}{ Participant B } \\
& Right & Left & & Right & Left \\
\hline Pushing force (max.) $[\mathrm{N}]$ & 6.7 & 7.37 & & 5.24 & 7.37 \\
Pushing force (ave.) $[\mathrm{N}]$ & 2.42 & 1.19 & & 0.55 & 4.87 \\
\hline
\end{tabular}

\section{Discussion of mutual response between user and robotic cart during operation}

When using our proposed mobile robotic cart, users hold the rigid handle directly. Therefore, the difference between the velocities of the user and the robot generates a mutual interference force during the operation of the prototype cart. We show the relationship between the reference and actual velocities for HOT patients in Fig. 19.

We found that periodic fluctuation occurs owing to the walking motion of the patients, as shown in Fig. 19, when the participant operated the prototype robotic cart. The velocities oscillate around the average speed of $0.2-$ $0.3 \mathrm{~m} / \mathrm{s}$, and the oscillation involves the mutual interference of the force which reduces the operability of the cart, describe above. Thus it is necessary to suppress the vibration force caused by the mutual interference to prevent the discomfort of the users.

To meet the requirement, we arrange the parameter $\mathrm{T}$ in the feedforward controller $C_{1}(s)$ for the users to adjust the start-up sensitivity of the moving velocity to suppress the vibration force caused by the mutual interference. Figure 20 shows a block diagram of the control system used to model the mechanism of mutual interference between the user and the prototype robotic cart. The interaction forces between the user and the prototype cart occur by the mechanism enclosed by the broken line in Fig. 20, which is added to the control system shown in
Fig. 9. In this control system, $C_{2}(s)$ is the velocity feedback controller for the control object $G(s)$ from the input $\hat{v}_{\text {ref }}$ to the output $v$, and the controller is designed to make the feedback system stable as described above. Therefore, the closed-loop transfer function from $\hat{v}_{\text {ref }}$ to $v$ becomes a stable transfer function.

Here we rewrite the system block diagram of Fig. 20 as shown in Fig. 21 to determine the mutual interference force. The control system in Fig. 21 can be interpreted as a force feedback control system between the pushing force of the user and the propulsion of the prototype robotic cart. As the transfer function of the closed-loop velocity controller in this system is stable, the characteristic of this force control system is determined by the combination of the robotic cart's dynamics and the feedforward controller $C_{1}(s)$.

We examine the effect of the feedforward controller $C_{1}$ $(s)$ on the properties of the force control system. We first examine the cutoff frequency, which indicates the startup response time of $C_{1}(s)$ when the HOT patients and elderly participants carried out the test. We then determined the principal frequency component of the control input (reference velocity) signal by FFT analysis when each participant operated the prototype robotic cart.

Figure 22 shows a scatter diagram of the cutoff frequency of $C_{1}(s)$ and the principal frequency component of the input signal of the HOT patients. In addition, we plot the results of a similar experiment on five elderly participants (female age 63, female age 63, male age 62, male age 69, female age 65) who are representative sample in Fig. 17b and five young participants who are normal males aged 22-24 for comparison.

The vertical axis in Fig. 22 shows the walking frequency of the participants, that is, the walking speed. The figure shows that the walking speed of the young and normal participants tended to be high compared with that of the 


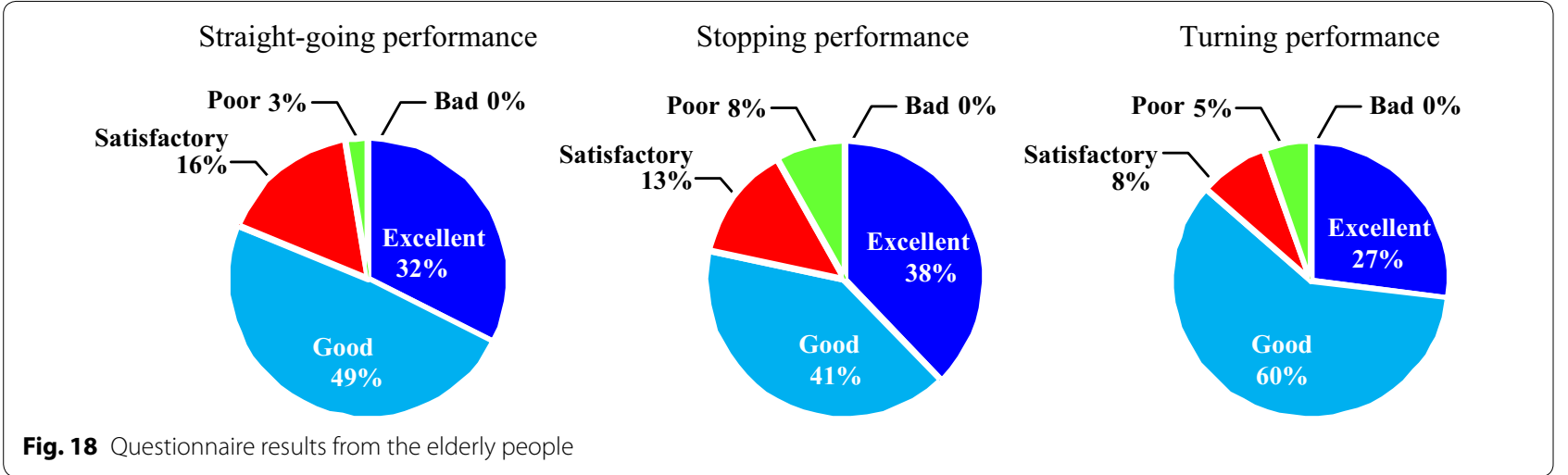
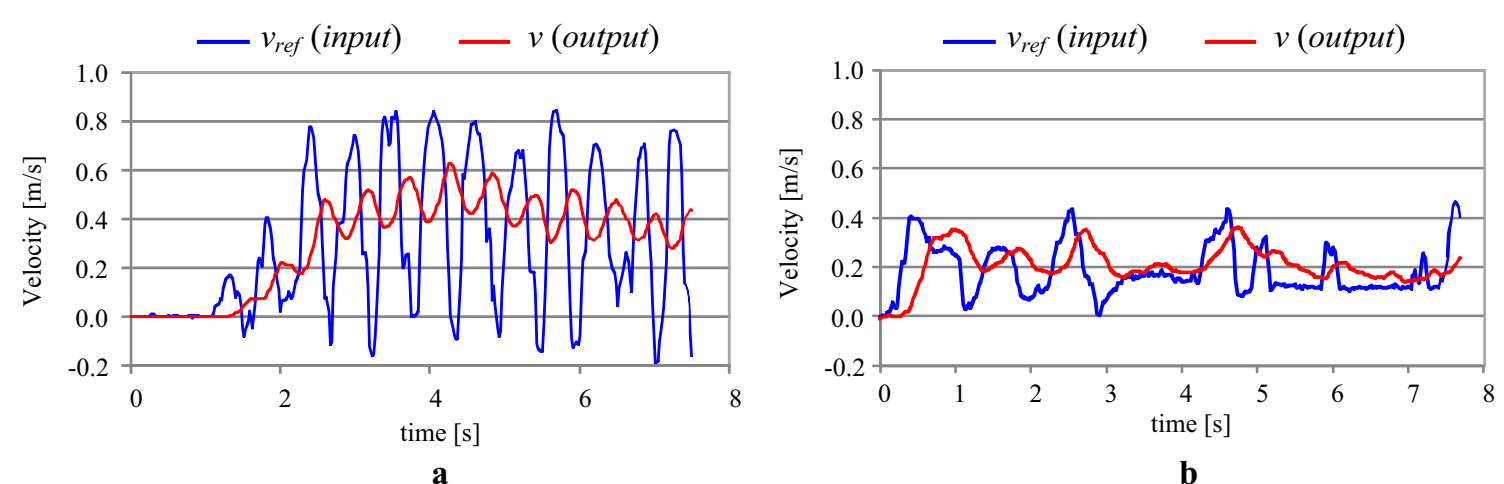

b

Fig. 19 Input-output response of velocity controller operated by the HOT patient. a Response between input $\left(v_{\text {ref }}\right)$ and output $(v)$ of participant A. b Response between input $\left(v_{\text {ref }}\right)$ and output $(v)$ of participant B

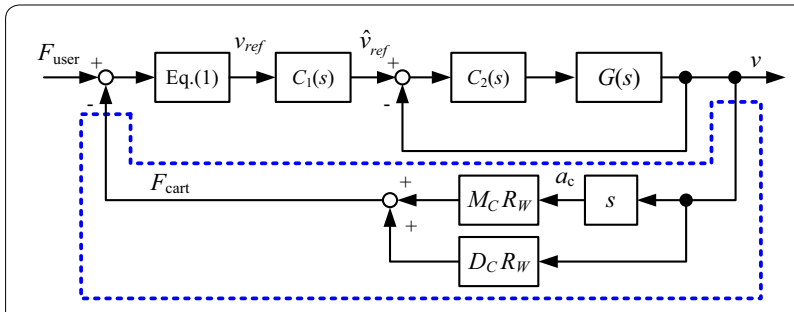

Fig. 20 Control system block diagram of our proposed mobile robot; which includes mutual interference between user and robotic cart where $M_{C}$ is mass of the mobile robotic cart, $D_{C}$ is coefficient of dynamic friction

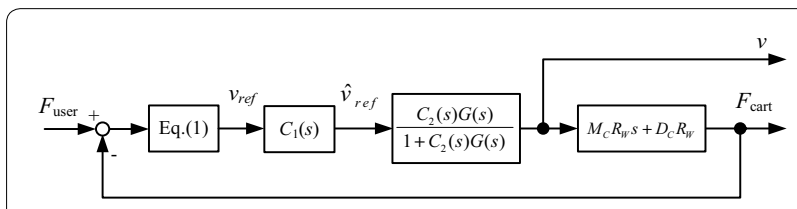

Fig. 21 Control system block diagram as a force control system

elderly and HOT patients. Also the cutoff frequencies of $C_{1}(s)$ are within the range of $0.2-0.6 \mathrm{~Hz}$ regardless of the type of participants as the adjusted results. This

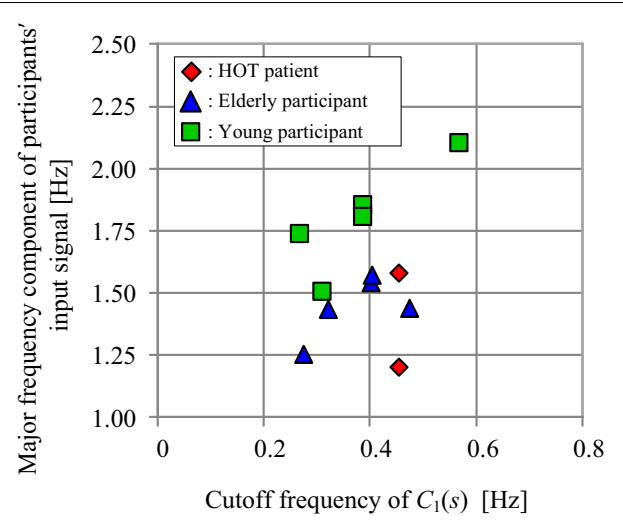

Fig. 22 Relations between patients' input signal and cutoff frequency of $C_{1}(s)$

result indicates that all the participants tended to adjust the parameter $\mathrm{T}$ of $C_{1}(s)$ to reduce the deviation of the steady force component. From the above results, our proposed control system is believed to effectively reduce mutual interference between the forces applied by the user and the prototype robotic cart. 


\section{Conclusions}

In this paper we proposed a mobile robotic cart similar to a wheeled walker that carries oxygen equipment to support HOT patients when they go out. We also proposed a new operation interface mechanism employing a rigid handle with several commonly used force sensors and a motion control system. Specifically, for the new operation interface, we proposed a new mechanical structure and force sensor arrangement that prevent erroneous operation due to grasping, twisting, and leaning on the handle. Then we proposed a two-degrees-of-freedom control system whose inputs are the pushing forces of the user and whose outputs are the velocities of the drive wheels of the robotic cart.

We then carried out several experiments by using a prototype robotic cart and showed the effectiveness of the power assistance for carrying the portable oxygen equipment. We also evaluated the control system by performing several experiments using the prototype mobile robotic cart and found that the feedforward controller in the two-degrees-of-freedom control system acted as a control element of the force control system between the user and the mobile robotic cart.

In future works, we will attempt to design a mobile robotic cart that is able to move in an outdoor environment and perform user evaluation tests. We also plan to solve these remaining problems with the system, with the aim of its early commercialization.

\section{Additional file}

Additional file 1. This video clip shows a scene of the user test in which HOT patients evaluated the operability of the robotic cart. The evaluation tests were carried out with an experimental assistant who took the participant's side and controlled a safety switch to prevent the emergency situation. The experiments are approved by the ethical committee of Tokyo Institute of Technology (approval number 2013002). We also obtained the consents of the participants for all experiments. The test was carried out in cooperation with the patient-based Kiyose-KIBOEN group in Tokyo on September 26, 2013.

\section{Authors' contributions \\ MI led and directed the research, proposed the concept of interface and con- trol system, designed the experiments, and analyzed the experimental data. NM designed the robotic cart in detail and performed the experiments and contributed to analyze the experimental data. GE proposed the concept of robotic cart, contributed to the design of the experiment with HOT patients, and submitted the proposal for ethical review. TT provided a medical review of the experiments. MO arranged the experiments with HOT patients. TK evaluated the robotic control system. All authors read and approved the final manuscript.}

\footnotetext{
Author details

${ }^{1}$ Department of Electro-Mechanical Engineering, Osaka Electro-Communication University, 18-8 Hatsu-cho, Neyagawa-shi, Osaka 572-8530, Japan. ${ }^{2}$ Department of Mechanical and Aerospace Engineering, Tokyo Institute of Technology, 2-12-1 Ookayama, Meguro-ku, Tokyo 152-8552, Japan.
}

${ }^{3}$ School of Medicine, Tokyo Women's Medical University, 8-1 Kawada-cho, Shinjuku-ku, Tokyo 162-8666, Japan. ${ }^{4}$ East Nagano Hospital, 2-477 Ueno, Nagano-shi 381-8567, Nagano, Japan. ${ }^{5}$ Department of Mechanical Systems Engineering, Okayama University of Science, 1-1 Ridai-cho, Kitaku, Okayama-shi 700-0005, Okayama, Japan.

\section{Acknowledgements}

The authors would like to express our deepest gratitude to the members and staff of Meeting for the Pulmonary Rehabilitation Studies in Hokushin, Hokushin Flying Disc Club and NPO Corporation J-BREATH for the questionnaire-based needs survey. The authors also would like to express our deepest gratitude to the members and staff of the patient-based group of respiratory involvement in Kiyose-KIBOEN and NPO Corporation Osaka Kohreisha Daigakko for the user test. The user test was also financially supported by the Association for Technical Aids (ATA).

\section{Competing interests}

The authors declare that they have no competing interests.

Received: 16 September 2015 Accepted: 22 June 2016

Published online: 11 July 2016

References

1. Gas Review Co. Ltd (2012) The number of home oxygen patients exceeds 160,000. Gas Medicina Gas Review 17:22 (in Japanese)

2. The white paper of home breathing care, Clinical Pulmonary Functions Committee of the Japanese Respiratory Society (2010) Questionnaires about daily life of HOT patients. pp 58-59

3. Iribe M, Matsuda H, Aizawa H, Endo G, Takubo T (2011) Study on a practical robotic follower to support daily life-a mobile robot development for home oxygen therapy patients with the 'Hyper Tether'. J Robot Mechatron 23(2):316-323

4. Tani A, Endo G, Fukushima EF, Hirose S, Iribe M, Takubo T (2011) Study on a practical robotic follower to support home oxygen therapy patientsdevelopment and control of a mobile platform. In: Proceedings of the 2011 IEEE/RSJ International Conference on Intelligent Robots and Systems, pp 2423-2429

5. Endo G, Allan B, lemura Y, Fukushima EF, Iribe M, Takubo T, Ohira M (2015) Mobile follower robot as an assistive device for home oxygen therapyevaluation of tether control algorithms. ROBOMECH J 2:6

6. Iribe M, Endo G, Maeta N, Onishi K, Ikeda R, lemura Y, Takubo T, Ohira M (2014) Practical assistive robot cart development to support hot patients. In: Proceedings of the ISCIE/ASME 2014 international symposium on flexible automation (ISFA2014). ISCIE/ASME, Hyogo, p 1165

7. Frizera A, Ceres R, Pons JL, Abellanas A, Raya R (2008) The smart walkers as geriatric assistive device. The SIMBIOSIS purpose. Gerontechnology (Valkenswaard) 7:108-115

8. Martins MM, Santos CP, Frizera-Neto A, Ceres R (2012) Assistive mobility devices focusing on smart walkers: classification and review. J Robot Auton Syst 60(4):548-562

9. Zhou W, Xu Li, Yang Jie (2010) An intent-based control approach for an intelligent mobility aid. In: Proceedings of 2 nd International Asia Conference on informatics in control, automation and robotics. IEEE, Wuhan, p 54-57

10. Chuy OY, Hirata Y, Wang Z, Kosuge K (2007) A control approach based on passive behavior to enhance user interaction. IEEE Transact Robot 23(5):899-908

11. Cortés U, Martínez-Velasco A, Barrué C, Martín X, Campana F, Annicchiarico R, Caltagirone C (2008) Towards an intelligent service to elders mobility using the i-Walker. In: Proceedings of AAAl fall symposia Al in eldercare: new solutions to old problems. AAAI, Washington, p 32-38

12. Shi F, Cao Q, Leng C, Tan H (2010) Based on force sensing-controlled human-machine interaction system for walking assistant robot. In: Proceedings of the 8th world congress on intelligent control and automation 2010. IEEE, Jinan, p 6528-6533

13. Horiuchi K, Aoki K (2003) Measurement of driving force of a rollator for aged people (Silver Car). Jpn J Ergon 39(1):38-41 (in Japanese) 